03,04

\title{
Влияние ионизирующего излучения на диэлектрические характеристики монокристаллов $\mathrm{Cu}_{2} \mathrm{ZnSn}\left(\mathrm{S}_{x} \mathrm{Se}_{1-x}\right)_{4}$
}

\author{
() В.Г. Гуртовой, А.У. Шелег \\ НПЦ НАН Беларуси по материаловедению, \\ Минск, Беларусь \\ E-mail: hurtavy@physics.by \\ (Поступила в Редакцию 28 июня 2016 г.)
}

Изучено влияние электронного облучения на проводимость и диэлектрические характеристики монокристаллов $\mathrm{Cu}_{2} \mathrm{ZnSnS}_{4}, \mathrm{Cu}_{2} \mathrm{ZnSnSe}_{4}$ и твердых растворов на их основе. Показано, что с увеличением дозы облучения значения диэлектрической проницаемости уменьшаются, а удельной электропроводности резко возрастают.

DOI: 10.21883/FTT.2017.02.44040.263

\section{1. Введение}

В настоящее время перспективными материалами для использования в качестве базовых слоев преобразователей солнечного излучения являются твердые растворы $\mathrm{Cu}(\mathrm{In}, \mathrm{Ga}) \mathrm{Se}_{2}, \mathrm{CuIn}(\mathrm{S}, \mathrm{Se})_{2}, \mathrm{CuGa}(\mathrm{S}, \mathrm{Se})_{2}$, которые, в отличие от кремния, являются прямозонными полупроводниками и обладают большим коэффициентом поглощения в видимой и ближней ИК-области спектра. Последние исследования этих систем показали, что, используя твердые растворы $\mathrm{Cu}(\mathrm{In}, \mathrm{Ga}) \mathrm{Se}_{2}$ в качестве базовой основы фотопреобразователей солнечного излучения, можно получить КПД 20.8-21.7\% [1,2]. Однако использовать эти материалы на практике не выгодно в силу высокой стоимости индия и галлия и ограниченности их земных запасов. Поэтому их начали заменять более доступными и дешевыми элементами. Четверные соединения $\mathrm{Cu}_{2} \mathrm{ZnSnS}_{4}$ и $\mathrm{Cu}_{2} \mathrm{ZnSnSe}_{4}$ в этом отношении представляют значительный интерес, так как они являются прямозонными полупроводниками, где дорогостоящие индий и галлий заменены на более распространенные и дешевые цинк и олово.

В последние годы в литературе появилось большое количество публикаций, посвященных как получению этих материалов и исследованию их физических свойств, так и их применению в солнечной энергетике [3-11]. Однако, несмотря на большое количество работ и быстрый прогресс в этом направлении, КПД солнечных элементов на основе $\mathrm{Cu}_{2} \mathrm{ZnSnSe}_{4}$ к настоящему времени получен на уровне $\sim 11.1-12.6 \%$ [12]. Причина низкой эффективности состоит в том, что существующие технологии не позволяют получать эти материалы в виде тонких пленок с высоким качеством структуры и необходимыми для солнечных элементов фотоэлектрическими свойствами. Кроме четверных соединений $\mathrm{Cu}_{2} \mathrm{ZnSnS}_{4}$ и $\mathrm{Cu}_{2} \mathrm{ZnSnSe}_{4}$ значительный интерес для солнечной энергетики представляют твердые растворы $\mathrm{Cu}_{2} \mathrm{ZnSn}\left(\mathrm{S}_{x} \mathrm{Se}_{1-x}\right)_{4}$. Изменяя их состав, можно получать материалы с непрерывно изменяющимися физическими свойствами, что дает возможность выбрать те составы, которые по своим характеристикам наиболее пригодны для использования на практике.

Поскольку эти материалы могут использоваться в качестве базовых слоев преобразователей солнечного излучения, возникает вопрос об их радиационной стойкости к ионизирующим излучениям. Поэтому целью данной работы было установление закономерностей изменения диэлектрических характеристик монокристаллов $\mathrm{Cu}_{2} \mathrm{ZnSn}\left(\mathrm{S}_{x} \mathrm{Se}_{1-x}\right)_{4}$ от температуры и дозы электронного облучения.

\section{2. Методика получения образцов}

Для синтеза четверных соединений $\mathrm{Cu}_{2} \mathrm{ZnSnS}_{4}$, $\mathrm{Cu}_{2} \mathrm{ZnSnSe}_{4}$ и твердых растворов $\mathrm{Cu}_{2} \mathrm{ZnSn}\left(\mathrm{S}_{x} \mathrm{Se}_{1-x}\right)_{4}$ использовали однотемпературный метод, обеспечивающий чистоту получаемого вещества и отсутствие потерь компонентов. Исходными веществами служили элементарные компоненты полупроводниковой чистоты: медь, цинк и олово чистоты $99.999 \%$, сера и селен марки ОСЧ. Синтез проводили в двойных кварцевых ампулах. Исходные компоненты в соотношениях, соответствующих формульному составу, в количестве $\sim 15 \mathrm{~g}$ загружали в ампулу, которую затем вакуумировали. Эту ампулу помещали в другую вакуумированную ампулу, которую помещали в вертикальную однозонную печь. Температуру в печи поэтапно с двухчасовой выдержкой поднимали до значений, на $20-30^{\circ} \mathrm{C}$ превышающих температуру плавления соединения либо температуры ликвидуса соответствующего состава твердого раствора. При достижении нужной температуры включали вибрационное перемешивание и выдерживали в течение $4 \mathrm{~h}$. Затем вибрацию отключали и уменьшали температуру со скоростью $5 \mathrm{~K} / \mathrm{h}$ до полного затвердения состава. Для гомогенизации полученных слитков твердых растворов проводили их изотермический отжиг в вакууме при $750^{\circ} \mathrm{C}$ в течение $300 \mathrm{~h}$. 
Монокристаллы $\mathrm{Cu}_{2} \mathrm{ZnSn}\left(\mathrm{S}_{x} \mathrm{Se}_{1-x}\right)_{4}$ выращивались методом химических газотранспортных реакций из синтезированных ранее поликристаллических слитков. В качестве газа-переносчика использовали элементарный йод марки В5. Синтезированные поликристаллы растирали в порошок и использовали в качестве исходного материала для газотранспорта. Ампулу с исходным материалом и йодом помещали в горизонтальную печь с двумя независимо регулируемыми зонами для создания необходимого градиента температур вдоль ампулы. Температуру в печи регулировали так, чтобы в зоне кристаллизации она была $\sim 700^{\circ} \mathrm{C}$, а в зоне реакции $780^{\circ} \mathrm{C}$. При этих условиях происходил рост монокристаллов в течение $\sim 8$ суток.

\section{3. Методика эксперимента}

Измерения диэлектрической проницаемости $(\varepsilon)$ и электропроводности $(\sigma)$ монокристаллических образцов проводили методом плоского конденсатора на монокристаллических пластинках с размерами $\sim 2 \times 5 \times 0.5 \mathrm{~mm}$ вдоль кристаллографического направления [001] с помощью цифрового измерителя Е7-20 на частотах измерительного поля $10^{3}-10^{6} \mathrm{~Hz}$ в температурном диапазоне 100-300 К. На образцы наносили омические серебряные контакты и помещали между металлическими прижимными контактами. Держатель с образцом экранировали латунным стаканом, на который через изоляционную прослойку из слюды наматывали нагреватель. Питание нагревателя осуществлялось постоянным током от стабилизированного источника. Температура контролировалась при помощи дифференциальной хромель-копелевой термопары и универсального цифрового вольтметра. Для измерений использовали метод непрерывного квазистатического нагревания со скоростью $\sim 0.5 \mathrm{~K} / \mathrm{min}$. Точность измерения температуры составляла $0.1-0.2 \mathrm{~K}$. Погрешность измерений диэлектрических характеристик составляла $\sim 0.5 \%$. Значения диэлектрической проницаемости и удельной электропроводности рассчитывались по формуле, соответствующей плоскопараллельному конденсатору.

Проводились исследования как необлученных образцов, так и облученных электронами с энергией $4 \mathrm{MeV}$ дозами $10^{15}$ и $10^{16} \mathrm{~cm}^{-2}$.

\section{4. Результаты исследований}

Исследованы диэлектрические характеристики полученных монокристаллов $\mathrm{Cu}_{2} \mathrm{ZnSnS}_{4}, \mathrm{Cu}_{2} \mathrm{ZnSnSe}_{4}$ и $\mathrm{Cu}_{2} \mathrm{ZnSn}\left(\mathrm{S}_{0.5} \mathrm{Se}_{0.5}\right)_{4}$ в интервале температур 100-300 K на различных частотах измерительного поля вдоль кристаллографического направления [001]. На рис. 1 и 2 приведены кривые температурных зависимостей относительной диэлектрической проницаемости и удельной электропроводности монокристаллов $\mathrm{Cu}_{2} \mathrm{ZnSn}\left(\mathrm{S}_{x} \mathrm{Se}_{1-x}\right)_{4}$ для $x=0.5$. На рис. 3 и 4 приведены кривые температурных зависимостей $\varepsilon$ и $\sigma$ на частоте $10^{5} \mathrm{~Hz}$

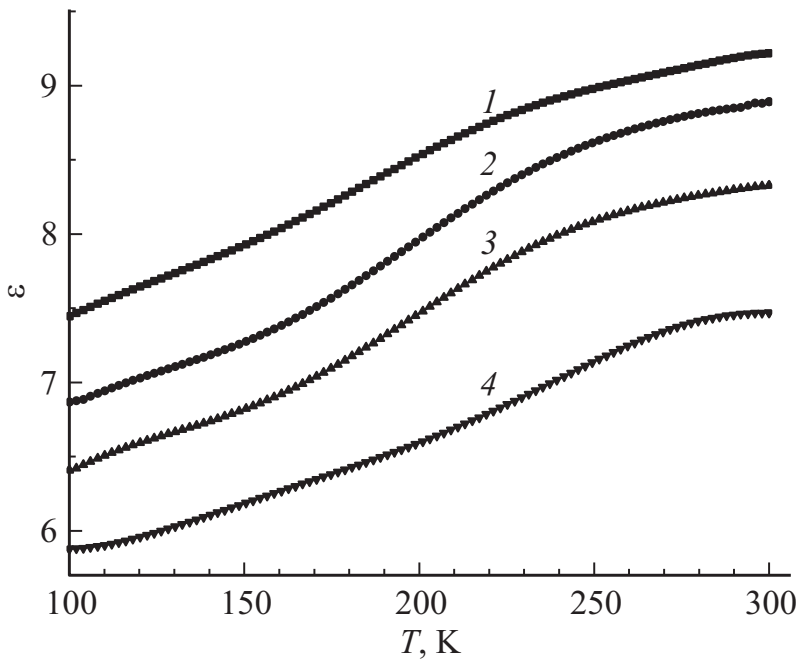

Рис. 1. Температурные зависимости диэлектрической проницаемости монокристаллов $\mathrm{Cu}_{2} \mathrm{ZnSn}\left(\mathrm{S}_{x} \mathrm{Se}_{1-x}\right)_{4}$ для $x=0.5$ на частотах: $1-10^{3} \mathrm{~Hz} ; 2-10^{4} \mathrm{~Hz} ; 3-10^{5} \mathrm{~Hz} ; 4-10^{6} \mathrm{~Hz}$.

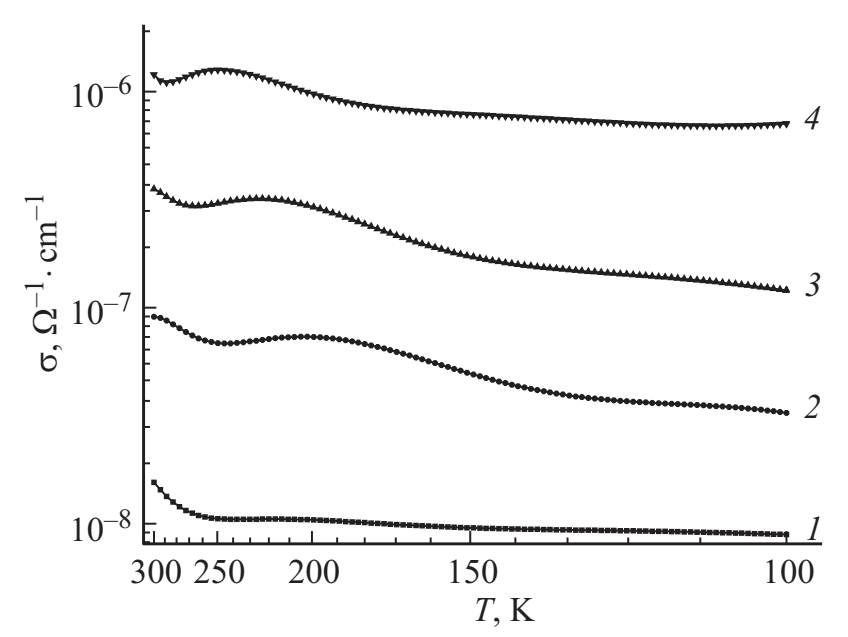

Рис. 2. Температурные зависимости удельной электропроводности монокристаллов $\mathrm{Cu}_{2} \mathrm{ZnSn}\left(\mathrm{S}_{x} \mathrm{Se}_{1-x}\right)_{4}$ для $x=0.5$ на частотах: $1-10^{3} \mathrm{~Hz} ; 2-10^{4} \mathrm{~Hz} ; 3-10^{5} \mathrm{~Hz} ; 4-10^{6} \mathrm{~Hz}$.

монокристаллов $\mathrm{Cu}_{2} \mathrm{ZnSn}\left(\mathrm{S}_{x} \mathrm{Se}_{1-x}\right)_{4}$ для различных $x$. Как видно из рисунков, значения $\varepsilon$ и $\sigma$ с ростом температуры увеличиваются. Это обусловлено увеличением концентрации свободных носителей зарядов (проявление полупроводниковых свойств) и ростом подвижности доменных границ (проявление сегнетоэлектрических свойств) в результате повышения температуры. Видно, что наблюдается значительная дисперсия диэлектрической проницаемости $\varepsilon$ и проводимости $\sigma$. С ростом частоты значения $\varepsilon$ уменьшаются (см. рис. 1), что обусловлено, как известно, релаксационными процессами, происходящими в высокочастотной области измерений. Электропроводность с ростом частоты увеличивается на несколько порядков (см. рис. 2). Такое поведение электрических свойств исследованных монокристаллов, также как и наличие максимумов на кривых $\sigma=f(T)$, 


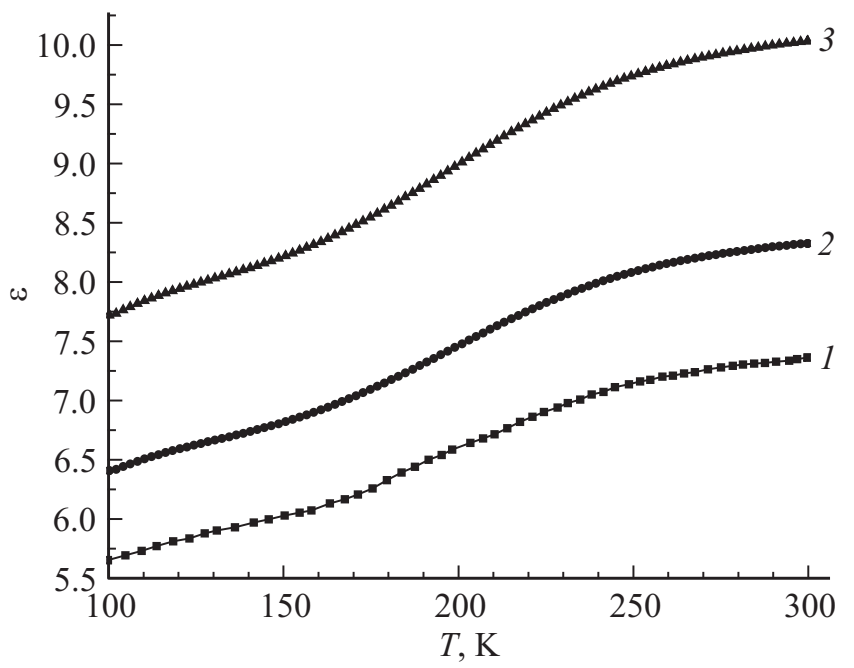

Рис. 3. Температурные зависимости диэлектрической проницаемости монокристаллов $\mathrm{Cu}_{2} \mathrm{ZnSn}\left(\mathrm{S}_{x} \mathrm{Se}_{1-x}\right)_{4}$ на частоте $10^{5} \mathrm{~Hz}: 1-x=1 ; 2-x=0.5 ; 3-x=0$.

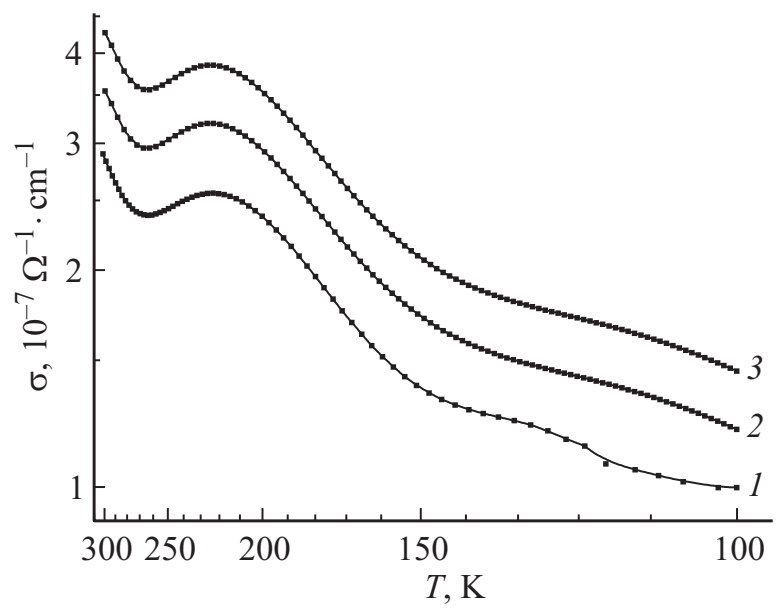

Рис. 4. Температурные зависимости удельной проводимости монокристаллов $\mathrm{Cu}_{2} \mathrm{ZnSn}\left(\mathrm{S}_{x} \mathrm{Se}_{1-x}\right)_{4}$ на частоте $10^{5} \mathrm{~Hz}$ : $1-x=1 ; 2-x=0.5 ; 3-x=0$.

по-видимому, связано с особенностями строения их кристаллической структуры, и как следствие сложным механизмом переноса заряда. Участки с разным наклоном на кривых $\sigma=f(T)$ указывают на наличие нескольких типов проводимости в этих кристаллах.

Из рис. 3 и 4 видно, что замещение серы на селен приводит к некоторому возрастанию значений диэлектрической проницаемости и проводимости. Полученные результаты по влиянию состава на диэлектрические свойства находятся в неплохом соответствии с данными [13], где из результатов оптических исследований определены значения высокочастотной диэлектрической проницаемости $\varepsilon_{\infty}$.

Изучены температурные зависимости диэлектрической проницаемости и проводимости монокристаллов твердых растворов $\mathrm{Cu}_{2} \mathrm{ZnSn}\left(\mathrm{S}_{x} \mathrm{Se}_{1-x}\right)_{4}$ в зависимости от дозы электронного облучения. Полученные монокристаллы подвергались облучению электронами с энергией $4 \mathrm{MeV}$ дозами $10^{15}$ и $10^{16} \mathrm{~cm}^{-2}$. На рис. 5 и 6 приведены температурные зависимости диэлектрической проницаемости $\varepsilon$ и проводимости $\sigma$ монокристаллов $\mathrm{Cu}_{2} \mathrm{ZnSnS}_{4}$ для различных доз облучения на частоте измерительного поля $10^{5} \mathrm{~Hz}$. Температурные зависимости для других составов и частот выглядят аналогично. Из рисунков видно, что облучение монокристаллов пучком электронов приводит к уменьшению значений диэлектрической проницаемости и значительному возрастанию электропроводности во всей исследованной области температур. Такое поведение значений $\varepsilon$ под воздействием облучения электронами может быть вызвано радиационно стимулированным старением образцов, связанным с

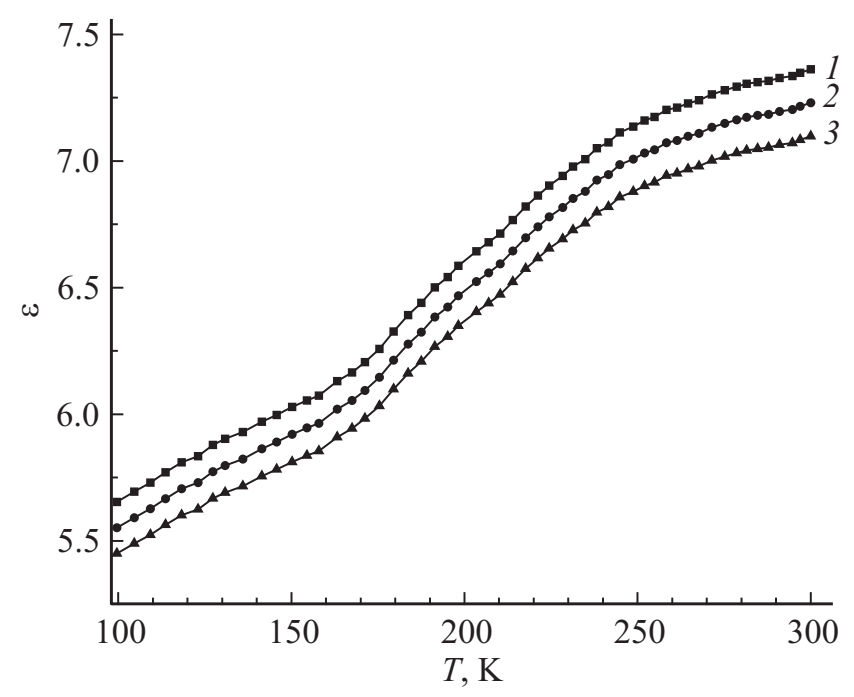

Рис. 5. Температурные зависимости диэлектрической проницаемости монокристаллов $\mathrm{Cu}_{2} \mathrm{ZnSnS}_{4}$ на частоте $10^{5} \mathrm{~Hz}$ для доз облучения: $1-0 \mathrm{~cm}^{-2} ; 2-10^{15} \mathrm{~cm}^{-2} ; 3-10^{16} \mathrm{~cm}^{-2}$.

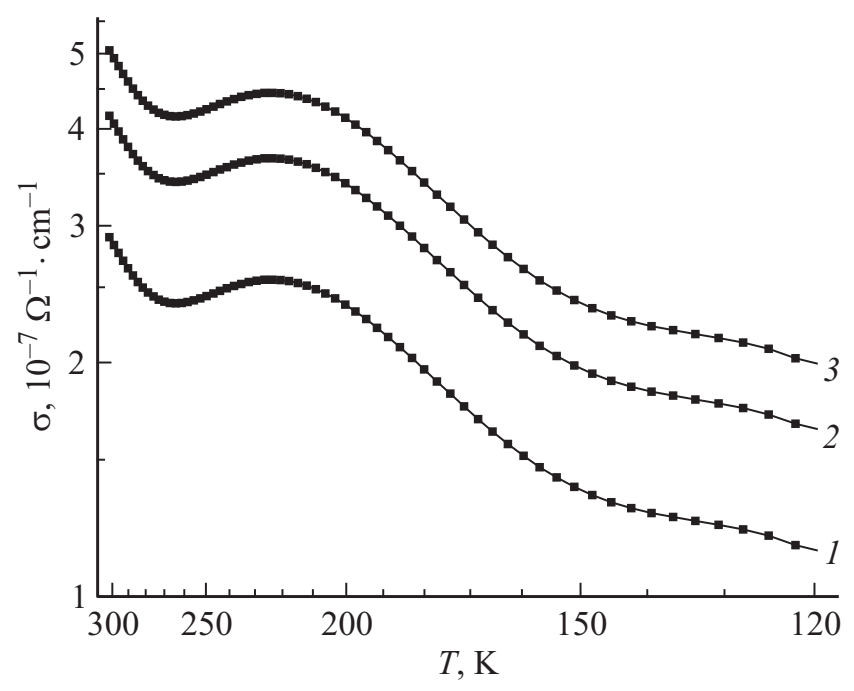

Рис. 6. Температурные зависимости удельной проводимости монокристаллов $\mathrm{Cu}_{2} \mathrm{ZnSnS}_{4}$ на частоте $10^{5} \mathrm{~Hz}$ для доз облучения: $1-0 \mathrm{~cm}^{-2} ; 2-10^{15} \mathrm{~cm}^{-2} ; 3-10^{16} \mathrm{~cm}^{-2}$. 
активизацией процесса миграции естественных дефектов под влиянием облучения, приводящие к стабилизации доменной структуры и снижению значений $\varepsilon$ [14]. Причиной роста значений электропроводности $\sigma$ монокристаллов соединений $\mathrm{Cu}_{2} \mathrm{ZnSn}\left(\mathrm{S}_{x} \mathrm{Se}_{1-x}\right)_{4}$ является, скорее всего, увеличение концентрации дефектов и, как следствие, возрастания вклада примесной проводимости.

\section{5. Заключение}

Проведены исследования влияния состава и дозы электронного облучения на монокристаллы четверных соединений $\mathrm{Cu}_{2} \mathrm{ZnSn}\left(\mathrm{S}_{x} \mathrm{Se}_{1-x}\right)_{4}$ с $x=0 ; 0.5 ; 1$.

Показано, что замещение серы на селен приводит к некоторому возрастанию значений диэлектрической проницаемости и проводимости.

Выявлено существенное влияние облучения электронами на электропроводность и диэлектрическую проницаемость исследованных монокристаллов. Увеличение дозы облучения приводит к уменьшению значений диэлектрической проницаемости и значительному возрастанию электропроводности во всей исследованной области температур.

\section{Список литературы}

[1] P. Jackson, D. Hariskos, R. Wuerz, W. Wischmann, M. Powalla. Phys. Status Solidi (RRL) - Rapid Res. Lett. 8, 3, 219 (2014).

[2] P. Jackson, D. Hariskos, R. Wuerz, O. Kiowski, A. Bauer, T.M. Friedlmeier, M. Powalla. Phys. Status Solidi (RRL) 9, 1, (2015).

[3] M. Grossberg, J. Krustok, J. Raudoja, T. Raadik. Appl. Phys. Lett. 101, 10, 102 102-1 (2012).

[4] R. Lydia, P. Sreedhara Reddy. J. Nano- Electron. Phys. 5, 3, 03017 (2013).

[5] F. Luckert, D.I. Hamilton, M.V. Yakushev, N.S. Beattie, G. Zoppi, M. Moynihan, I. Forbes, A.V. Karotki, A.V. Mudryi, M. Grossberg, J. Krustok, R.W. Martin. Appl. Phys. Lett. 99, 6, 062104 (2011).

[6] M. León, S. Levcenko, R. Serna, A. Nateprov, G. Gurieva, J.M. Merino, S. Schorr, E. Arushanov. Mater. Chem. Phys. 141, 1, 58 (2013).

[7] A. Singh, S. Singh, S. Levcenko, T. Unold, F. Laffir, K.M. Ryan. Angewandte Chem. Int. Ed. 52, 35, 9120 (2013).

[8] T.K. Todorov, J. Tang, S. Bag, O. Gunawan, T. Gokmen, Y. Zhu, D.B. Mitzi. Adv. Energy Mater. 3, 1, 34 (2013).

[9] А.У. Шелег, В.Г. Гуртовой, А.В. Мудрый, В.Д. Живулько, М.Я. Валах, В.А. Юхимчук, И.С. Бабичук, Н. Хіе, Е. Saucedo. Журн. прикл. спектроскопии 81, 5, 704 (2014).

[10] А.У. Шелег, В.Г. Гуртовой, А.В. Мудрый, М.Я. Валах, B.A. Юхимчук, И.С. Бабичук, M. León, R. Caballero. ФТП 48, 10, 1332 (2014).

[11] А.У. Шелег, В.Г. Гуртовой, В.А. Чумак. Кристаллография 60, 5, 826 (2015).

[12] W. Wang, M.T. Winkler, O. Gunawan, T. Gokmen, T.K. Todorov, Y. Zhu, D.B. Mitzi. Adv. Energy Mater. 4, 7, 36 (2014).

[13] M. Kumar, C. Persson. Int. J. Theor. Appl. Sci. 5, 1, 1 (2013).

[14] Е.В. Пешиков. Радиационные эффекты в сегнетоэлектриках. Фан. Ташкент (1986). с. 18. 